\title{
Immunodépression des Mères au VIH et Morphométrie des Nourrissons à Abidjan
}

\author{
Kouadio Kouakou Jérôme, (Docteur, Maître-Assistant) \\ Institut des Sciences Anthropologiques de Développement (ISAD), \\ Université Félix Houphouët-Boigny d'Abidjan Cocody (UFHB)
}

Doi: 10.19044/esj.2019.v15n3p28～URL:http://dx.doi.org/10.19044/esj.2019.v15n3p28

\section{Résumé}

Le profil morphologique des nourrissons serait, entre autres, fonction de facteurs spécifiques dont l'état biophysiologique de la mère. L'effet de celui-ci sur le développement corporel du nourrisson ne semble suffisamment pas être étudié. Le présent travail a pour objectif d'examiner l'influence de l'immunodépression de la mère au vih sur la croissance corporelle du nourrisson de cette dernière. Il porte sur 128 nourrissons âgés de 1 à 24 mois dont 57 nés de mères sérodéprimées et 71 nés de mères non sérodéprimées. Le profil nutritionnel du nourrisson est jugé à travers le poids et la stature de ce dernier. La balance électronique et la toise staturale sont utilisées pour mesurer respectivement ces paramètres corporels. La sérologie des mères est explorée en considérant les résultats des tests sérologiques réalisés par ces mères durant les consultations prénatales. Les résultats montrent que le poids et la stature moyens des enfants nés de mères sérodéprimées ne s'écartent pas de ceux de leurs homologues nés de mères qui ne sont pas sérodéprimées. La sérodépression de la mère au vih est loin d'influencer significativement le développement physique des enfants à bas âge.

Mots clés: VIH, Mère, Développement physique, Nourrisson, Côte d'Ivoire 


\title{
Immunocompromision of Mothers with HIV and Morphometry of Nurslings in Abidjan
}

\author{
Kouadio Kouakou Jérôme, (Docteur, Maître-Assistant) \\ Institut des Sciences Anthropologiques de Développement (ISAD), \\ Université Félix Houphouët-Boigny d'Abidjan Cocody (UFHB)
}

\begin{abstract}
The morphological profile of nurslings is, among other things, a function of specific factors including the biophysiological status of the mother. The effect of this on infant body development does not seem to be sufficiently studied. The purpose of this paper is to examine the influence of mother's immunodepression on HIV on her nurslig's body growth. It covers 128 nurslings aged 1 to 24 months of whom 57 are born to serocompromised mothers and 71 are born to mothers who are not serocompromised mothers. The nutritional profile of the nursling is judged by the weight and stature of the nursling. The electronic scale and the height gauge are used to measure these bodily parameters respectively. The serology of the mothers is explored by considering the results of the serological tests carried out by these mothers during the prenatal consultations. The results show that the average weight and height of children born to serocompromised mothers do not differ from those of their counterparts born to mothers who are not serocompromised. The serocompromision of the mother to hiv is far from significantly influencing the physical development of young children.
\end{abstract}

Keywords: HIV, Mother, Physical Development, Nursling, Ivory Coast

\section{Introduction}

L'enfance est une période durant laquelle l'individu serait soumis, entre autres, à des aliments spécifiques nécessaires à sa croissance physique. Celle-ci serait synonyme de ce que l'on appelle le profil nutritionnel de l'enfant. Celui-ci se réfère au produit de l'interaction complexe entre son équipement biologique propre et les stimulations bio-alimentaires dont il bénéficie (Allen, 1994).

Cette relation entre l'adaptation corporelle de l'enfant et le cadre bioalimentaire est d'autant étroite que, selon Brocklehurst \& French (1998), l'aliment ingéré par l'enfant, à cet âge, est le liquide physiologique secrété par les mamelons de sa génitrice, c'est-à-dire le lait maternel. Alors que certaines mères le disposeraient à souhait à leur nourrisson, d'autres, dont le sang est en 
mauvais état, notamment celles infectées au vih, ne sauraient allaiter leur progéniture.

Le développement corporel du nourrisson pourrait être influencé par le statut immunologique de sa mère au vih. Celui-ci renvoie à l'indice de présence du virus de l'immunodéficience humaine (vih) ou non dans le sang qui caractérise un individu (Educalingo, 2018). Selon que le sang de la mère contient le vih, elle est catégorisée comme ayant un statut sérologique positif. La mère présente un statut sérologique négatif, si elle ne porte pas ce virus dans son sérum sanguin (République Démocratique du Congo, 2008).Ainsi, une mère séropositive ne pourrait offrir à son nourrisson un allaitement naturel nécessaire à une bonne adaptabilité. Il en résulte généralement chez le nourrisson, privé de cette alimentation nutritive et protectrice, une anémie et un état de maigreur (Konaté, 2009)[6].

En revanche, lorsqu'une mère est séronégative, c'est-à-dire ayant un sérum sanguin dépourvu de vih, elle est dans les conditions appropriées pour offrir une alimentation optimale, en l'occurrence du lait maternel à son nourrisson. Le faisant, la mère procure à l'organisme de son nourrisson des substances nutritives, telles les vitamines, les sels minéraux, des protéines, des glucides, des sucres assimilables, des graisses nécessaires au bon développement physique de l'enfant (Programme national nutrition santé, 2009).

Dans cette perspective, Diouf et al. (2016) ont étudié les risques de transmission mère-enfant du vih et le devenir nutritionnel des nourrissons dans l'option B+. Sous cet angle, Kaaya et al. (2016) ont examiné la relation entre la dépression maternelle et la malnutrition infantile chez les femmes vivant avec le vih.

Il semble que la relation entre la sérologie de la mère et le profil nutritionnel de l'enfant est loin d'être suffisamment exploré. L'objectif de la présente est d'examiner la relation entre la sérologie de la mère et le profil nutritionnel du nourrisson.

\section{Méthodologie}

\section{1. Matériel}

La population des femmes enceintes séropositives au vih, vivant en Côte d'Ivoire, est estimée à 11182 femmes, soit 1,2\% de la proportion des femmes enceintes (Ministère de la Santé et de l'Hygiène Publique, 2016). Celles-ci, âgées de 18 à 43 ans, résident, pour la plupart, à Abidjan (Institut National de la Statistique, 2012). Elles y reçoivent régulièrement un traitement antirétroviral (ARV) durant la grossesse et après l'accouchement dans les centres de Prévention de Transmission Mère-Enfant (PTME) qui leur sont proposés. Elles y vont en compagnie de leurs nouveau-nés ou nourrissons pour 
permettre à ceux-ci de bénéficier des soins nutritionnels, car, selon Mutombo et al. (1996), le taux de malnutrition chez ces enfants avoisine $12 \%$.

Dans ce cas et conformément aux indications de Schwartz (1963), la taille de l'échantillon à observer pourrait être estimée à partir de ce taux, en considérant la formule suivante. Taille de l'échantillon $(\mathrm{N}): \mathrm{N}==\mathrm{t}^{2} \times \mathrm{p}(1-\mathrm{p})$ $/ \mathrm{m}^{2} . \mathrm{N}=$ taille d'échantillon requise, $\mathrm{t}=$ niveau de confiance à $95 \%$ (valeur type de 1,96), $\mathrm{p}=$ prévalence estimative de la malnutrition dans la zone d'étude $(12 \%$ ou 0,12$), \mathrm{m}=$ marge d'erreur à $5 \%$ (valeur type de 0,05 ). $\mathrm{N}=1.96^{2} \mathrm{x}$ $0,12(1-0,12) / 0,05^{2}=163$ sujets.

L’indisponibilité d'une base de données portant sur les nourrissons nés de mères séropositives et le refus de plusieurs mères ne permettent ni une sélection probabiliste des dyades ni de retenir la taille de l'échantillon précédemment indiquée. Par conséquent, un échantillon par convenance de dyades mère-enfant, séropositives ou séronégatives, d'origine socioéconomique défavorisée, est constitué, après avoir obtenu le consentement éclairé de ces dernières. Cet échantillonnage (au jugé) s'est effectué dans le district sanitaire Abobo Est et dans 1'Hôpital Général d'Abobo Nord Abidjan, choisis aléatoirement parmi plusieurs (environ 164 PTME). L'échantillon retenu, à l'issue de ce procédé, comprend 128 nourrissons de 0 à 24 mois dont 57 nourrissons nés de mères séropositives et 71 nourrissons nés de mères séronégatives.

\subsection{Méthodes}

L'examen de la relation entre la sérodépression de la mère au vih et le développement corporel des enfants de 0 à 24 mois est une étude de type explicatif transversal. Il est réalisé de janvier à mars 2016 suivant différentes opérations observationnelles. La première, qui est une ré-enquête doublée d'un pré-test, a consisté à soumettre à 08 dyades mère-enfant, sélectionnées systématiquement dans l'enceinte du centre de santé communautaire d'Abobo Banco Sud, durant 10 jours, un questionnaire de 72 items et des mensurations corporelles. Cette primo-observation, a permis d'améliorer et de valider le questionnaire d'échantillonnage et les outils de mesure pour le profil physique. Ainsi, l'enquête proprement dite s'est effectuée auprès de dyades mère-enfant fréquentant l'Hôpital général d'Abobo Nord, sélectionné aléatoirement. Ainsi, l'on a, d'abord, soumis à 136 dyades mère-enfant (62 séropositives, 74 séronégatives), choisies au hasard, dans cet hôpital, un questionnaire de 61 items et des mensurations corporelles portant sur le poids, la stature des nourrissons et / ou des mères.

Le questionnaire a examiné les caractéristiques sociodémographiques, notamment la situation familiale, le rang de naissance de l'enfant dans la fratrie, la situation économique; les connaissances sur les pratiques alimentaires (mode alimentaire, durée de ce mode, la date de sevrage des 
nourrissons) et l'aliment de complément chez les enfants de 6 à 24 mois. Aussi, les informations concernant la date de naissance et le sexe, le poids de naissance, le terme, le statut vaccinal, le statut de supplémentation en vitamine A et le suivi biologique des enfants (réalisation de la PCR après un mois), ontelles sont vérifiées dans le carnet d'accouchement de la mère et le carnet de santé / vaccination des enfants.

Ainsi, les données relatives à la mesure poids et la stature actuelle des nourrissons sont collectées, selon les indications de l'OMS (2008), par des enquêteurs (internes de médecine, le personnel habituel du service de vaccination) à qui un rappel sur les techniques de mesures est préalablement fait au cours de séances d'entrainement. Les enfants sont pesés dévêtus au moyen d'un pèse-bébé de type SECA avec une précision de $10 \mathrm{~g}$. Le poids des enfants de moins de 2 ans, non coopératifs, est déterminé par la différence entre le poids du couple mère-enfant et le poids de la mère dont la mesure est réalisée en utilisant un pèse-personne de fonction tare et de précision $100 \mathrm{~g}$.

La stature des enfants est mesurée par deux enquêteurs en utilisant une toise staturale en position horizontale. Pendant cet exercice, l'opérateur allonge l'enfant sur la toise, les pieds du côté du curseur. L'enfant doit être au milieu de la toise. L'assistant prend la tête de l'enfant entre ses mains, au niveau, des oreilles et la maintient bien en place contre la partie fixe de la toise, l'enfant regardant droit devant lui. L'opérateur place ses mains au-dessus des chevilles de l'enfant ou sur les genoux. En maintenant bien les jambes, il place le curseur contre la plante des pieds de l'enfant en s'assurant que ceux-ci ne sont pas décollés. Il effectue alors la lecture.

Les enquêteurs s'assurent, avant chaque pesée, que l'aiguille de la balance est remise à zéro. De même, le poids et la stature sont mesurés pour chaque enfant, avant qu'il ne soit vacciné. Cette opération, qui a duré 3 mois, a permis de considérer les données de 128 dyades mère-enfant, comparables au plan socio-démographique et économique. La saisie et le nettoyage des données sont faits en se servant des logiciels Nutrisurvey Anthro v3.2.2 (2005. exe); Excel et SPSS (version 23.0) pour Windows.

Ensuite, la distribution de ces données corporelles structurées est examinée statistiquement en appliquant le test de Kolmogorov Smirnov. Les résultats de ce contrôle montrent que les données pondérales et staturales ne suivent pas une distribution normale ( $p$-value $=0,092 ; p$-value $=0,165$ ). Les mesures obtenues sont présentées sous forme de scores moyens, concernant le poids et la stature, considérés séparément.

Enfin, l'analyse statistique, enfin, de ces données s'est effectuée au moyen du test non paramétrique kruskal-wallis, pour tenir compte de la non normalité de la distribution des données. L'emploi de ce test se révèle nécessaire pour vérifier la relation supposée entre le statut 
d'immunodépression de la mère et la croissance corporelle des nourrissons. Le seuil, à partir duquel cette dernière est significative, est fixé à 0,05 .

\section{Résultats}

La question examinée porte sur le développement corporel du nourrisson en lien avec le statut au virus d'immunodéficience humaine (vih) de la mère de celui-ci. Les observations, faites auprès d'enfants de 1 à 24 mois nés de mères de statut sérologique différent, permettent d'apporter quelques éléments de réponse à cette préoccupation.

Chez les enfants âgés d'un à six mois (1 à 6 mois), ceux nés de mères de statut immuno-non-déprimées au vih présentent un poids $(5,73 \mathrm{~kg} \pm 1,07)$ et une stature $(59,40 \mathrm{~cm} \pm 3,86)$ moyens statiquement superposables à ceux des enfants nés de mères immunodéprimées au vih (poids $=4,67 \mathrm{~kg} \pm 1,36$, pvalue $=0,014$, et stature $=57,47 \mathrm{~cm} \pm 7,15$, p-value $=0,162$ ) (confère le tableau 1).

Tableau 1: Comparaison de la stature et du poids moyens des enfants de [1-6] mois selon le Statut de la mère au Virus d'Immunodéficience Humaine SVIH.

\begin{tabular}{lllll}
\hline SVIH & Sexe & N & Poids $(\mathbf{m} \pm \boldsymbol{\sigma})$ & Stature $(\mathbf{m} \pm \boldsymbol{\sigma})$ \\
\hline Positif & Fille & 09 & $4,24 \mathrm{~kg} \pm 1,23$ & $54,78 \mathrm{~cm} \pm 7,12$ \\
\cline { 2 - 5 } & Garçon & 06 & $5,32 \mathrm{~kg} \pm 1,38$ & $61,50 \mathrm{~cm} \pm 5,44$ \\
\hline Kruskul-wallis & & & $\mathrm{p}=0,099 \mathrm{NS}$ & $\mathrm{p}=0,036 \mathrm{~S}$ \\
\hline Négatif & Fille & 17 & $5,49 \mathrm{~kg} \pm 1,05$ & $58,82 \mathrm{~cm} \pm 3,78$ \\
\cline { 2 - 5 } & Garçon & 14 & $6,04 \mathrm{~kg} \pm 1,06$ & $60,15 \mathrm{~cm} \pm 3,97$
\end{tabular}

kruskul-wallis

$\mathrm{p}=0,194 \mathrm{NS}$

$\mathrm{p}=0,401 \mathrm{NS}$

kruskul-wallis général

$\mathrm{p}=0,014 \mathrm{~S}$

$\mathrm{p}=0,162 \mathrm{NS}$

N: Effectif des sujets; S: Différence Significative; NS: Différence Non Significative; p: pvalue

Des constats similaires sont faits, lorsque l'on compare le poids et la stature moyens des autres tranches d'âge. Ainsi, l'on note chez les enfants âgés de six à douze mois (6 à 12 mois) nés de mères immuno-non-déprimées au vih un poids $(7,74 \mathrm{~kg} \pm 1,61)$ et une stature $(67,96 \mathrm{~cm} \pm 4,90)$ moyens analogues à ceux de leurs homologues nés de mères de statut sérologique positif (poids $=7,60 \mathrm{~kg} \pm 1,06$ et stature $=66,55 \mathrm{~cm} \pm 4,18$ ) (p-values respectives $0,572 \mathrm{et}$ 0,533 ) (confère le tableau 2).

Tableau 2: Comparaison de la stature et du poids moyens des enfants de [6-12] mois à partir de l'indice Poids/Age selon le SVIH.

\begin{tabular}{lllll}
\hline SVIH & Sexe & $\mathbf{N}$ & Poids $(\mathbf{m} \pm \boldsymbol{\sigma})$ & Stature $(\mathbf{m} \pm \boldsymbol{\sigma})$ \\
\hline Positif & Fille & 14 & $7,42 \mathrm{~kg} \pm 0,90$ & $65,78 \mathrm{~cm} \pm 4,10$ \\
\cline { 2 - 5 } & Garçon & 14 & $7,78 \mathrm{~kg} \pm 1,22$ & $67,32 \mathrm{~cm} \pm 4,26$ \\
\hline Kruskul-wallis & & & $\mathrm{p}=0,260 \mathrm{NS}$ & $\mathrm{p}=0,269 \mathrm{NS}$ \\
\hline \multirow{2}{*}{ Négatif } & Fille & 14 & $7,60 \mathrm{~kg} \pm 1,79$ & $67,78 \mathrm{~cm} \pm 5,79$ \\
\cline { 2 - 5 } & Garçon & 14 & $7,87 \mathrm{~kg} \pm 1,45$ & $68,14 \mathrm{~cm} \pm 4,03$ \\
\hline
\end{tabular}




\begin{tabular}{lll}
\hline Kruskul-wallis & $\mathrm{p}=0,448 \mathrm{NS}$ & $\mathrm{p}=1,000 \mathrm{NS}$ \\
\hline Kruskul-wallis général & $\mathrm{p}=0,572 \mathrm{NS}$ & $\mathrm{p}=0,533 \mathrm{NS}$ \\
\hline
\end{tabular}

N: Effectif des sujets; NS: Différence Non Significative; p: p-value

L'égalité du développement des deux groupes d'enfants semble être constante, puisque les résultats, observés chez les nourrissons âgés de 12 à 24 mois, ne s'écartent pas de ceux indiqués précédemment. Ainsi, l'on note chez les enfants nés de mères immunodéprimées, un poids $(9,02 \mathrm{~kg} \pm 1,24)$ et une stature $(73,68 \mathrm{~cm} \pm 3,16)$ moyens équivalents aux poids $(8,05 \mathrm{~kg} \pm 1,20)$ et à la stature $(71,63 \mathrm{~cm} \pm 3,93)$ moyens de leurs homologues nés de mère qui ne sont pas immunodéprimées ( $\mathrm{p}$-value: 0,027 pour le poids et 0,215 pour la stature (confère le tableau 3).

Tableau 3: Comparaison de la stature et du poids moyens des enfants de[12-24] mois à partir de l'indice Poids/Age selon le SVIH.

\begin{tabular}{lllll}
\hline SVIH & Sexe & $\mathbf{N}$ & Poids $(\mathbf{m} \pm \boldsymbol{\sigma})$ & Stature $(\mathbf{m} \pm \boldsymbol{\sigma})$ \\
\hline Positif & Fille & 05 & $8,74 \mathrm{~kg} \pm 1,21$ & $73,70 \mathrm{~cm} \pm 3,07$ \\
\cline { 2 - 5 } & Garçon & 09 & $9,16 \mathrm{~kg} \pm 1,30$ & $73,67 \mathrm{~cm} \pm 3,39$ \\
\hline Kruskul-wallis & & & $\mathrm{p}=0,789 \mathrm{NS}$ & $\mathrm{p}=0,947 \mathrm{NS}$ \\
\hline Négatif & Fille & 07 & $7,68 \mathrm{~kg} \pm 0,54$ & $7,68 \mathrm{~cm} \pm 2,32$ \\
\cline { 2 - 5 } & Garçon & 05 & $8,55 \mathrm{~kg} \pm 1,73$ & $70,60 \mathrm{~cm} \pm 5,67$ \\
\hline Kruskul-wallis & & & $\mathrm{p}=0,371 \mathrm{NS}$ & $\mathrm{p}=0,683 \mathrm{NS}$ \\
\hline Kruskul-wallis $\mathrm{p}=0,027 \mathrm{~S}$ & & & $\mathrm{p}=0,215 \mathrm{NS}$ \\
\hline
\end{tabular}

N: Effectif des sujets; NS: Différence Non Significative; p: p-value

De tels résultats pourraient trouver une explication dans des observations empiriques.

\section{Discussion}

L'examen de l'influence du statut au vih de la mère sur le profil morphologique de l'enfant constitue l'objet d'étude du présent travail. Les résultats des observations réalisées dans cette perspective, auprès de nourrissons âgés de 1 à 24 mois, montrent que les nourrissons nés de mères immuno-non-déprimées présentent une croissance corporelle qui ne diffère statistiquement pas de celle de leurs pairs de mères immunodéprimées (confère les tableaux 1, 2 et 3 ). Le statut au vih de la mère au vih n'exerce suffisamment pas un effet significatif sur le développement physique des nourrissons.

Plusieurs facteurs permettent d'expliquer ces résultats. L'un des éléments pouvant être à l'origine de la non influence de la sérodépression la mère sur le profil développemental de l'enfant serait l'administration des ARV à des mères immunodéprimées au vih dès la première consultation prénatale. En effet, selon Diouf et al. (2016), la prise des ARV au cours de la grossesse et de l'accouchement réduit efficacement la transmission du virus de la mère à l'enfant, en diminuant la réplication du virus chez la mère d'une part, en assurant une prophylaxie chez le nourrisson pendant et après son exposition 
au virus de l'autre (United Nations International Children's Emergency Fund (Unicef, 2015).

Ainsi, dans ces conditions, l'enfant né d'une mère séropositive recevrait la même qualité alimentaire que son homologue né de mère qui n'est pas immunodéprimée. Ces résultats sont semblables à ceux de Lawson et al. (2015). Ces derniers, dans leur étude menée au Togo, n'ont pas trouvé de relation statistiquement significative entre le statut au vih de la mère et la morphologie des enfants.

L'égalité entre les deux groupes d'enfants ne varie pas selon le sexe et l'âge (confère les tableaux 1, 2 et 3). La non différence de développement physique observé, selon le sexe, dans le présent travail, aurait pour source la standardisation des caractéristiques biologiques et physiques des enfants à travers l'action correctrice des centres de PTME.

A l'analyse, la croissance morphologique des nourrissons nés de mère de statut au vih différent pourrait être liée à des caractéristiques bioindividuelles, alimentaires et socio-environnementales de la dyade mèrenourrisson. Certaines, que sont le potentiel physio-intrinsèque de la mère, de l'enfant et l'alimentation proposée à ce dernier, exercent un effet direct sur le développement morphologique. D'autres, en l'occurrence le niveau socioéconomique de la mère ou de la famille, influent indirectement sur la croissance corporelle. Ces mécanismes ne sauraient, bien qu'ils sous-tendent les résultats de 1'étude réalisée, être donc extrapolables.

\section{Conclusion}

Le présent travail se pose la question du développement morphologique des nourrissons nés de mères immunodéprimées au vih. L'hypothèse qui sous-tend ce questionnement est l'idée selon laquelle l'immunodépression de la mère au vih a un effet négatif sur le développement corporel du nourrisson de cette dernière. L'expérimentation sur terrain relative à cette préoccupation a consisté à comparer le poids et la stature des nourrissons, filles et garçons, dont l'âge varie entre [1-6 mois [, [6-12 mois [ et [12-24 mois], de mères sérodéprimées à ceux de leurs pairs de mères non immunodéprimées. Les résultats obtenus montrent que les nourrissons de mères sérodéprimées au vih présentent un poids et une stature qui correspondent avec ceux de leurs homologues de mères qui ne sont pas sérodéprimées au vih. L'immunodépression au vih de la mère ne créerait suffisamment pas de différence entre les nourrissons, du point de vue du développement corporel.

\section{Remerciements}

L'auteur de l'étude est reconnaissant à l'égard des responsables du district sanitaire Abobo Est, ceux de l'Hôpital général d'Abobo Nord pour 
avoir accepté que l'enquête se déroule au sein de leur établissement. Il remercie également le personnel du centre de Prévention de Transmission Mère-Enfant de l'Hôpital général d'Abobo Nord pour son soutien à la collecte des données. L'auteur adresse aussi ses remerciements aux mères et à leurs enfants (sujets), qui ont permis la réalisation de l'observation factuelle.

\section{Conflit d'interêts}

Il n'existe aucun conflit.

\section{References:}

1. Allen, L. H. (1994). Nutritional influences on linear growth: a general review. European Journal of Clinical Nutrition, 48 (Suppl. 1), 75-89.

2. Brocklehurst, P., \& French, R. (1998). The association between maternal HIV infection and perinatal out come: a systematic review of the literature and meta-analysis. British Journal of Obstetrics and Gynaecology, 105 (8), 836-848.

3. Diouf, J. B., Diallo, D., Sylla, A., Mbaye, N., Ouattara, B., \& Ndiaye, O. (2016). Devenir sérologique et nutritionnel des nourrissons nés de mères séropositives suivis dans l'option $\mathrm{B}+$ à Guédiawaye [Serological and nutritional outcome of infants born to HIV positive mothers undergoing option B + therapy in Guédiawaye].The Pan $\begin{array}{llll}\text { African medical journal, } 224 . & \end{array}$ doi:10.11604/pamj.2016.25.224.10441

4. Educalingo (2008). Sérologique. En ligne https://educalingo.com/fr/dic-fr/serologique, consulté le 05 novembre 2018.

5. Institut National de la Statistique (INS, 2012). Enquête démographique et de santé et à indicateurs multiples 2011-2012. Abidjan: INS.

6. Kaaya, S., Garcia, M. E., Li, N., Lienert, J., Twayigize, W., Spiegelman, D., \& Smith, F. M. C. (2016). Association of maternal depression and infant nutritional status among women living with HIV in Tanzania. Maternal \& Child Nutrition, 12 (3), 603-613. doi: 10.1111/mcn.12154.

7. Konaté, S (2009). Former les membres d'une communauté en Déviance Positive, c'est participé à l'épanouissement du bien-être de ses enfants, lutter contre la pauvreté et la dépendance à l'aide extérieur. Bougouni: Manuel de formation en nutrition déviance positive des rélais et partenaires (agents de terrain).

8. Lawson-Evi, K., Djadou, K. E., Tsolenyanu, E., Bakondé, B., AmeliHouado, N., Gbadoé, A.,... Atakouma, D. Y. (2015). Etat nutritionnel des enfants de moins de 5 ans apparemment sains reçus pour 
vaccination à Lomé (Togo) selon les nouvelles normes de l'OMS. Revue Cames Santé, 3 (2), 61-65.

9. Ministère de la Santé et de l'Hygiène Publique (MSHP, 2016). Rapport Annuel sur la Situation Sanitaire en Côte d'Ivoire. Abidjan: MSHP.

10. Mutombo, T., Keusse, J., \& Sangaré, A. (1996). Sida et malnutrition en milieu pédiatrique semi-rural expérience de l'hôpital protestant de Dabou en Côte d'ivoire. Médecine d'Afrique Noire, 43 (2), 73-77.

11. Organisation Mondiale de la Santé (2008). Cours de formation sur l'évaluation de la croissance de l'enfant. Genève: OMS.

12. Programme national Nutrition santé (2009). Le guide de l'allaitement maternel. Paris: Institut national de prévention et d'éducation pour la santé (INPES).

13. République Démocratique du Congo (2008). Loi $\mathrm{n}^{\circ}$ 08/011 du 14 juillet 2008 portant protection des droits des personnes vivant avec le VIH/SIDA et des personnes affectées. Journal officiel de la République Démocratique du Congo, 14, 9-16.

14. Schwartz, C. E. D. (1963) Méthodes statistiques à l'usage des médecins et biologistes. Paris: Flammarion Médecine Sciences.

15. United Nations International Children's Emergency Fund (Unicef, 2015). A Business Case for Option B and B+ to eliminate mother to child transmission of VIH. New York: Unicef. 